PRAMANA - JOURNAL OF PHYSICS

PUBLISHER: SPRINGER (GERMANY); IMPACT FACTOR: 0.692; ISSN : 0304-4289

ACCEPTED OCTOBER $15^{T H} 2016$ (IN PRESS)

\title{
PHYSICAL HYDRODYNAMIC PROPULSION MODEL STUDY FOR CREEPING VISCOUS FLOW THROUGH A CILIATED POROUS TUBE
}

\author{
Noreen Sher Akbar ${ }^{1}$, Adil Wahid Butt ${ }^{1}$, Dharmendra Tripathi ${ }^{2}$ and O. Anwar Bég ${ }^{3}$ \\ ${ }^{1}$ DBS\&H, CEME, National University of Sciences and Technology, Islamabad, Pakistan. \\ ${ }^{2}$ Department of Mechanical Engineering, Manipal University, Jaipur-303007, India. \\ ${ }^{3}$ Fluid Mechanics, Bio-Propulsion and Nanosystems, Aeronautical and Mechanical Engineering, \\ University of Salford, Newton Building, UG17, The Crescent, Salford, M54WT, England, UK.
}

\begin{abstract}
The present investigation focuses on a mathematical study of creeping viscous flow induced by metachronal wave propagation in a horizontal ciliated tube containing porous media. Creeping flow limitations are imposed i.e. inertial forces are small compared with viscous forces and therefore very low Reynolds number $(R e<<1)$ is taken into account. The wavelength of metachronal wave is also considered as very large for cilia movement. The physical problem is linearized and exact solutions are developed for the differential equation problem. Mathematica software is used to compute and illustrate numerical results. The influence of slip parameter and Darcy number on velocity profile, pressure gradient and trapping of bolus are discussed with the aid of graphs. It is found that with increasing magnitude of slip parameter the trapped bolus inside the streamlines increases in size. The study is relevant to biological propulsion of medical micro-machines in drug delivery.
\end{abstract}

Keywords: Viscous fluid; Ciliated tube; Porous medium; Darcy number; Permeability; Low Reynolds number; Hydrodynamic slip; Biomimetic propulsion.

\section{Nomenclature}

a radius of tube

$c$ wave speed

Da Darcy number

$F$ flow rate in moving frame

$\bar{h}$ height of tube

$K$ biological medium permeability

$\bar{P}$ pressure in the moving frame

$\bar{p}$ pressure in the fixed frame

$\triangle P$ pressure rise

$Q$ flow rate in the fixed frame 


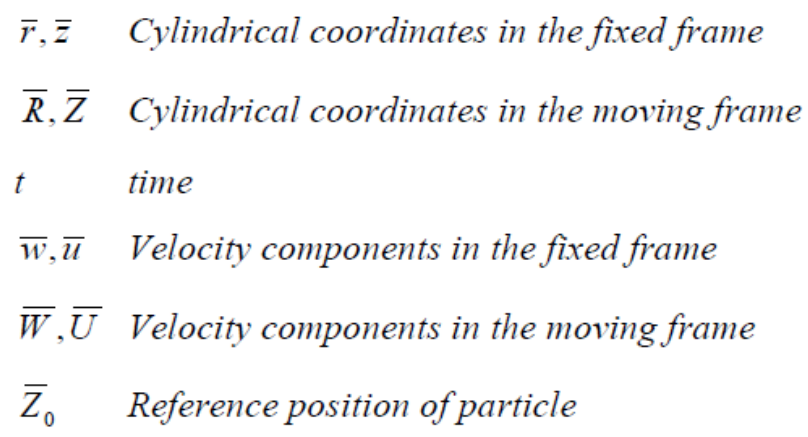

Greek

$\lambda \quad$ wavelength

$\alpha \quad$ eccentricity of the elliptical motion

$\alpha_{1} \quad$ hydrodynamic slip parameter

$\beta \quad$ wave number

$\varepsilon \quad$ cilia length

$\lambda \quad$ wavelength

$\mu \quad$ dynamic viscosity of fluid

$\rho \quad$ density offluid

\section{INTRODUCTION}

Biological propulsion is increasingly attracting the interest of engineers owing to diverse applications in medicine, aerospace, naval and even geological systems. Many mathematical models have been proposed for different organisms and micro-organisms at different length scales and Reynolds numbers. A most lucid review of the subject has been given by Wu [1] in which 18 orders of magnitude have been identified for the range of flows studied from microscopic organisms to large marine mammals (e.g. the blue whale). At the microscopic scale and in particular in the context of embryological systems, cilia and flagellar propulsion are of great interest. This area of biological hydrodynamic propulsion has also motivated significant interest for many decades, as elaborated by Wu et al. [2]. Cilia and flagella lengths may span from a few microns to more than $2 \mathrm{~mm}$ in the case of some insect sperm flagella. Hence, cilia and flagella essentially have no difference, although they have been ascribed different 
terminologies prior to a proper biological examination of their structures. Cilia have been established as beating with a whip-like asymmetric mechanism which comprises both an effective stroke and a recovery stroke. Moreover, when many cilia function collectively, fluid dynamic interactions may induce beating out-of-phase, and this manifests in the generation of metachronal waves, and exacerbated hydrodynamics flow. The specific metachrony is termed symplectic (or antiplectic) when the metachronal wave is in the same (or opposite) direction as the effective stroke. These features have recently attracted attention in biomimetics and bioinspired engineering systems, notably in medical micro-swimmers which offer tremendous potential in nano-medicine, drug-delivery and so forth, as described by Feng and Cho [3]. Commonly, cells own one or two long flagella. Conversely ciliated cells have many short cilia. For instance, the mammalian spermatozoa has a single flagellum, the unicellular green alga Chlamydomonas has two flagella, and the unicellular protozoan Paramecium is covered with a few thousand cilia, these are used for both locomotion and nutrition. In mammals, many epithelial cells are ciliated in order to sweep materials across the tissue surface. For instance, considerable numbers of cilia (more than $107 / \mathrm{mm}^{2}$ ) cover the surfaces of mammalian respiratory passages (the nose, pharynx, and trachea), here they dislodge and expel particulate matter that collects in the mucus secretions of these tissues. The motion of cilia and flagella is controlled by the Stokes equations (linear) with no-slip boundary conditions on their surfaces and vanishing fluid disturbance at infinity [4]. The structure of cilia, factors which affect cilia activities, movement of cilia and flagella and the coordination of the beating of cilia have been addressed analytically by Sleigh [5]. Lardner and Shack [6] examined cilia effects on transport flow rates in the ductus efferentes of the male reproductive tract. A mathematical model to represent the microstructure of ciliated organisms was developed by Blake [7]. In continuation of the studies on cilia movement and its importance in various fields of research, Wu [8] theoretically investigated the fluid mechanism of cilia motion. Brennen [9] presented an oscillatory thin shear layer (boundary layer) theory for cilia movement. Sleigh and Aiello [10] studied water movement by cilia. Agarwal and Uddin [11] investigated the fluid flow with variable viscosity by cilia transport; Blake [12] developed a spherical envelope approach for cilia motion. Miller [13] focused on the movement of Newtonian fluids sustained by mechanical cilia oscillation; Barton and Raynor [14] presented an analytical approach for cilia induced mucous flow; Smith et 
al. [15] addressed viscoelastic fluid flow effects on cilia transport. Dauptain et al. [16] studied computationally the hydrodynamics of cilia movement from a fluid-structure interaction (FSI) viewpoint, specifically simulating ctenophore Pleurobrachia pileus marine micro-organisms (quasi-spherical geometry) for multiple flexible cilia with 3-dimensional models. Khaderi and Onck [17] presented three dimensional computations for cilia movement. Khaderi et al. [18] further studied the non-reciprocal motion due to beating of artificial cilia. Another interesting communication by Khaderi et al. [19] reports on magnetically-actuated artificial cilia for microfluidic propulsion.

The above biological propulsion simulations were restricted to purely fluid media. In recent years significant interest has also developed in transport phenomena in biological porous media. Many organs in the human body comprise permeable materials including kidneys, lungs, tissue and the human skin [20]. Porous media hydrodynamics further arises in circulation of cerebrospinal fluid in the brain. In biomedical engineering, flow in porous media is integral to the fabrication of artificial physiological organs including artificial kidneys, gastric tract and lungs [21]. Almost invariably, biological materials (tissue etc) are heterogeneous and exhibit strongly anisotropy. These complex constitutive properties originate from the cellular microstructure. Although intrinsically the porous media arising in different organs of the body are anisotropic, approximations can be made to simulate transport through them. A popular approach is the Darcy model which has been used for microvasculature via networks of onedimensional segments, whereas the capillary bed is often approximated as a spatially-averaged Darcy compartment. Darcy's law and its modification assumes that the rate at which a fluid flows through a permeable substance per unit area is equal to the product of the permeability (a property of the material through which the fluid is flowing) and the pressure drop per unit length of flow, divided by the viscosity of the fluid. A porous medium is generally defined in continuum mechanics [22] as a medium which contains a number of small holes distributed throughout the matter. Khaled and Vafai [23] have shown that convective flow models for porous media are accurate for modelling hemodynamics in tumours and muscles and in particular mimick quite well the impeded transport of physiological fluids in vessels clogged with fatty cholestoral plaques and clots. Interstitial fluid mechanics also lends itself well to porous media transport since the tissue may be approximated as a medium of dispersed cells 
separated by connective voids, the latter permitting percolation of nutrients, minerals and other substances to cells within the tissue. Darcy's law however neglects the friction within the fluid and exchange of momentum between the fluid and solid phases. Modifications of Darcy models have therefore been developed for some time. A good example of flow with boundary condition at the interfaces of a porous medium was presented by Saffman [24]. Most physiological vessels feature porous media characteristics and considering this fact many works have been reported in literature. An interesting transport problem in porous media (of the moving boundary type) is peristalsis, and arises in intestinal fluid dynamics. Representative studies in this area have been communicated by for example Miyamoto et al. [25] who studied the two-dimensional laminar flow in a circular porous tube and considering a small water absorption or secretion in the intestinal perfusion experiment whereas Jeffrey et al. [26] discussed the flow fields generated by peristaltic reflex in isolated guinea pig ileum. Elshehawey et al. [27] developed another model for peristaltic transport through an asymmetric porous medium channel and focused the application to intra uterine fluid motion in a sagittal cross-section of the uterus. Other recent investigations on peristaltic flow of Newtonian fluid and non-Newtonian fluids have been presented and span many multi-physical aspects of the subject including power law fluids [28], magnetohydrodynamics [29] and Maxwell rheological pumping through porous media [30].

A careful inspection of the scientific literature has revealed however that the creeping flow induced by metachronal wave propagations of viscous fluid in a porous ciliated tube is yet to be studied mathematically in the literature. Porous media offer a well-tested mechanism for flow control in hydrodynamic propulsion. The present work aims to therefore address this topic for the first time. Analytical solutions are developed for the linear partial differential equations generated. Linearization is achieved by neglecting inertial forces relative to viscous forces, using the low Reynolds number approximation from hydrodynamic lubrication theory. Numerical results are presented graphically and the influence of key geometric and hydromechanical parameters (e.g. Darcy number) on transport characteristics is elaborated in detail. The simulations presented herein further elucidate the mechanism of creeping viscous flow induced by cilia motion and are relevant to gastric fluid mechanics and also biomimetic devices exploiting surface modifications for optimized propulsion [31-35]. 


\section{MATHEMATICAL MODEL}

Let us consider incompressible Newtonian flow in a ciliated tube with hydrodynamic slip at the wall. The tube interior wall is ciliated with metachronal waves and the flow is generated due to the collective beating of cilia. We select a cylindrical coordinate system $(\bar{R}, \bar{Z})$, where the $\bar{Z}$ axis is orientated along the center line of the tube and $\bar{R}-$ axis is normal to it. Cilia deform in a wave-like fashion with a propagation velocity, $c$, along the wall of the outer tube. Keeping in view the geometry of the metachronal wave pattern, it is assumed that the envelope of cilia tips can be expressed mathematically in the following form:

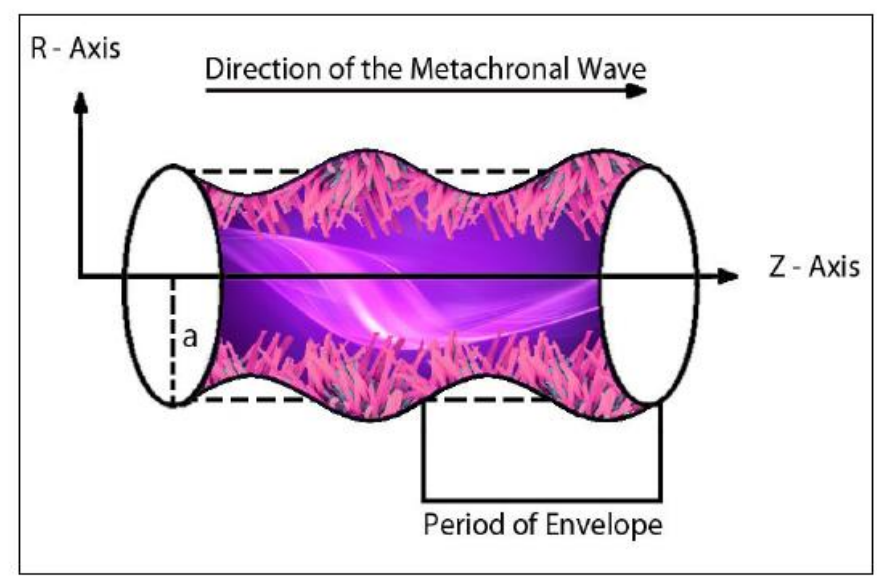

Fig. 1. Geometry of the problem

Fig. 1: Schematic of the biological propulsion problem

The envelops of the cilia tips can be expressed mathematically $[1,9]$ as follows:

$$
\begin{aligned}
& \bar{R}=\bar{H}=\bar{f}(\bar{Z}, \bar{t})=a+a \varepsilon \cos \left(\frac{2 \pi}{\lambda}(\bar{Z}-c \bar{t})\right), \\
& \bar{Z}=\bar{g}\left(\bar{Z}, \bar{Z}_{0}, \bar{t}\right)=a+a \varepsilon \alpha \sin \left(\frac{2 \pi}{\lambda}(\bar{Z}-c \bar{t})\right),
\end{aligned}
$$

where $a$ denotes the mean radius of the tube, $\varepsilon$ is the non-dimensional measure with respect to the cilia length, $\lambda$ and $c$ are the wavelength and wave speed of the metachronal wave respectively, $\bar{Z}_{0}$ denotes the reference position of the particle and $\alpha$ is a measure of the eccentricity of the elliptical motion. The velocities of the transport fluid are essentially those 
induced by the cilia tips, and may be defined as:

$$
\begin{aligned}
& \left.\bar{W}=\frac{\partial \bar{Z}}{\partial \bar{t}}\right)_{\bar{z}_{0}}=\frac{\partial \bar{g}}{\partial \bar{t}}+\frac{\partial \bar{g}}{\partial \bar{Z}} \frac{\partial \bar{Z}}{\partial \bar{t}}=\frac{\partial \bar{g}}{\partial \bar{t}}+\frac{\partial \bar{g}}{\partial \bar{Z}} \bar{W}, \\
& \left.\bar{U}=\frac{\partial \bar{R}}{\partial \bar{t}}\right)_{\bar{z}_{0}}=\frac{\partial \bar{f}}{\partial \bar{t}}+\frac{\partial \bar{f}}{\partial \bar{Z}} \frac{\partial \bar{Z}}{\partial \bar{t}}=\frac{\partial \bar{f}}{\partial \bar{t}}+\frac{\partial \bar{f}}{\partial \bar{Z}} \bar{W} .
\end{aligned}
$$

Using Eqn. (2) in Eqn. (1), we get

$$
\begin{aligned}
& \bar{W}=\frac{-\frac{2 \pi}{\lambda}\left[\varepsilon \alpha a c \cos \left(\frac{2 \pi}{\lambda}\right)(\bar{Z}-c \bar{t})\right]}{\left[1-\frac{2 \pi}{\lambda}\left\{\varepsilon \alpha a \cos \left(\frac{2 \pi}{\lambda}\right)(\bar{Z}-c \bar{t})\right\}\right]}, \\
& \bar{U}=\frac{\frac{2 \pi}{\lambda}\left[\varepsilon a c \sin \left(\frac{2 \pi}{\lambda}\right)(\bar{Z}-c \bar{t})\right]}{\left[1-\frac{2 \pi}{\lambda}\left\{\varepsilon \alpha a \cos \left(\frac{2 \pi}{\lambda}\right)(\bar{Z}-c \bar{t})\right\}\right]} .
\end{aligned}
$$

In the fixed coordinates $(\bar{R}, \bar{Z})$, the flow in the tube is unsteady. It becomes steady in a wave frame $(\bar{r}, \bar{z})$ moving with the same speed as the wave moves in the $\bar{Z}$-direction. The governing equations for the viscous flow through porous medium in moving frame is expressed as:

Continuity equation:

$$
\frac{1}{\bar{R}} \frac{\partial(\bar{R} \bar{U})}{\partial \bar{R}}+\frac{\partial \bar{W}}{\partial \bar{Z}}=0
$$

R-direction momentum equation:

$$
\begin{aligned}
\rho\left[\frac{\partial \bar{U}}{\partial \bar{t}}+\bar{U} \frac{\partial \bar{U}}{\partial \bar{R}}+\bar{W} \frac{\partial \bar{U}}{\partial \bar{Z}}\right]= & -\frac{\partial \bar{P}}{\partial \bar{R}}+\mu \frac{\partial}{\partial \bar{R}}\left[2 \frac{\partial \bar{U}}{\partial \bar{R}}\right]+\mu \frac{2}{\bar{R}}\left(\frac{\partial \bar{U}}{\partial \bar{R}}-\frac{\bar{U}}{\bar{R}}\right) \\
& +\mu \frac{\partial}{\partial \bar{Z}}\left[\left(\frac{\partial \bar{U}}{\partial \bar{R}}+\frac{\partial \bar{W}}{\partial \bar{Z}}\right)\right],
\end{aligned}
$$

\section{Z-direction momentum equation:}

$$
\begin{aligned}
\rho\left[\frac{\partial \bar{W}}{\partial \bar{t}}+\bar{U} \frac{\partial \bar{W}}{\partial \bar{R}}+\bar{W} \frac{\partial \bar{W}}{\partial \bar{Z}}\right]= & -\frac{\partial \bar{P}}{\partial \bar{Z}}+\mu \frac{\partial}{\partial \bar{Z}}\left[2 \frac{\partial \bar{W}}{\partial \bar{Z}}\right]+\mu \frac{1}{\bar{R}} \frac{\partial}{\partial \bar{R}}\left[\bar{R}\left(\frac{\partial \bar{U}}{\partial \bar{Z}}+\frac{\partial \bar{W}}{\partial \bar{R}}\right)\right] \\
& -\frac{\mu}{K} \bar{W}
\end{aligned}
$$

The transformations between the two frames are:

$$
\bar{r}=\bar{R}, \bar{z}=\bar{Z}-c \bar{t}, \bar{u}=\bar{U}, \bar{w}=\bar{W}-c, \bar{p}(\bar{z}, \bar{r})=\bar{P}(\bar{Z}, \bar{R}, \bar{t})
$$

The governing equations for the regime can be written via these transformations as follows: 


$$
\begin{gathered}
\frac{1}{\bar{r}} \frac{\partial(\bar{r} \bar{u})}{\partial \bar{r}}+\frac{\partial \bar{w}}{\partial \bar{z}}=0, \\
\rho\left[\bar{u} \frac{\partial \bar{u}}{\partial \bar{r}}+\bar{w} \frac{\partial \bar{u}}{\partial \bar{r}}\right]=-\frac{\partial \bar{P}}{\partial \bar{r}}+\mu \frac{\partial}{\partial \bar{r}}\left[2 \frac{\partial \bar{u}}{\partial \bar{r}}\right]+\mu \frac{2}{\bar{r}}\left(\frac{\partial \bar{u}}{\partial \bar{r}}-\frac{\bar{u}}{\bar{r}}\right) \\
+\mu \frac{\partial}{\partial \bar{z}}\left[\left(\frac{\partial \bar{u}}{\partial \bar{r}}+\frac{\partial \bar{w}}{\partial \bar{z}}\right)\right], \\
\rho\left[\bar{u} \frac{\partial \bar{w}}{\partial \bar{r}}+\bar{w} \frac{\partial \bar{w}}{\partial \bar{z}}\right]=-\frac{\partial \bar{P}}{\partial \bar{z}}+\mu \frac{\partial}{\partial \bar{z}}\left[2 \frac{\partial \bar{w}}{\partial \bar{z}}\right]+\mu \frac{1}{\bar{r}} \frac{\partial}{\partial \bar{r}}\left[\bar{r}\left(\frac{\partial \bar{u}}{\partial \bar{z}}+\frac{\partial \bar{w}}{\partial \bar{r}}\right)\right] \\
-\frac{\mu}{K}(\bar{w}+c),
\end{gathered}
$$

Corresponding boundary conditions are as defined in Ellahi et al .[31], Nadeem and Sadaf [35].

$$
\begin{aligned}
\frac{\partial \bar{w}}{\partial \bar{r}} & =0, \quad \text { at } \quad r=0, \\
w & =-c-c \frac{2 \pi \varepsilon \alpha \beta \cos (2 \pi z)}{1-2 \pi \varepsilon \alpha \beta \cos (2 \pi z)}-\frac{K}{\alpha_{1} *} \frac{\partial \bar{w}}{\partial \bar{r}}, \quad \text { at } r=\bar{h}(z) .
\end{aligned}
$$

Where $c \frac{2 \pi \varepsilon \alpha \beta \cos (2 \pi z)}{1-2 \pi \varepsilon \alpha \beta \cos (2 \pi z)}$ is cilia factor.

Introducing the following non-dimensional variables, which are defined in the nomenclature:

$$
r=\frac{\bar{r}}{a}, \quad z=\frac{\bar{z}}{\lambda}, w=\frac{\bar{w}}{c}, u=\frac{\lambda \bar{u}}{a c}, p=\frac{a^{2} \bar{p}}{c \lambda \mu}, \beta=\frac{a}{\lambda}, D_{a}=\frac{K}{a^{2}}, \alpha_{1}=\frac{\alpha_{1} *}{a} .
$$

Making use of these variables in Eqns. $(9,10)$, and using the assumptions of low Reynolds number and long wavelength, the non-dimensional governing equations can be written as:

$$
\begin{gathered}
\frac{\partial p}{\partial r}=0, \\
\frac{d p}{d z}=\frac{1}{r} \frac{\partial}{\partial r}\left(r \frac{\partial w}{\partial r}\right)-\frac{1}{D_{a}}(w+1),
\end{gathered}
$$

The non-dimensional boundary conditions on the ciliated porous walls are prescribed as: 


$$
\begin{aligned}
& \frac{\partial w}{\partial r}=0, \quad \text { at } \quad r=0, \\
& w=-1-\frac{2 \pi \varepsilon \alpha \beta \cos (2 \pi z)}{1-2 \pi \varepsilon \alpha \beta \cos (2 \pi z)}-\frac{\sqrt{D_{a}}}{\alpha_{1}} \frac{\partial w}{\partial r}, \quad \text { at } r=h(z)=1+\varepsilon \cos (2 \pi z) .
\end{aligned}
$$

\section{ANALYTICAL SOLUTIONS}

Integrating Eqn. (13) and using boundary conditions (14 a, b), axial velocity is obtained as:

$$
w(r, z)=-1-D_{a} \frac{d p}{d z}+\frac{\alpha_{1}\left(D_{a} \frac{d p}{d z}-\frac{2 \pi \varepsilon \alpha \beta \cos (2 \pi z)}{1-2 \pi \varepsilon \alpha \beta \cos (2 \pi)}\right) I_{0}\left(\frac{r}{\sqrt{D_{a}}}\right)}{I_{1}\left(\frac{h}{\sqrt{D_{a}}}\right)-I_{0}\left(\frac{h}{\sqrt{D_{a}}}\right)},
$$

The volumetric flow rate $(F)$ is defined as:

$$
F=2 \pi \int_{0}^{h} r w d r
$$

Integrating the above expression for flow rate, we have:

$$
\frac{d p}{d z}=\frac{\frac{I_{1}\left(\frac{h}{\sqrt{D_{a}}}\right)\left((2 \pi \varepsilon \alpha \beta \cos (2 \pi z)-1)\left(F+\pi h^{2}\right)-2 \pi \alpha_{1} \sqrt{D_{a}} h n\right)}{2 \pi \varepsilon \alpha \beta \cos (2 \pi z)-1}-\left(F+\pi h^{2}\right) I_{0}\left(\frac{h}{\sqrt{D_{a}}}\right)}{\pi D_{a} h\left(h I_{0}\left(\frac{h}{\sqrt{D_{a}}}\right)-\left(h-2 \alpha_{1} \sqrt{D_{a}}\right) I_{1}\left(\frac{h}{\sqrt{D_{a}}}\right)\right)}
$$

Flow rate in moving and fixed frames are related as follows:

$$
Q=F+\frac{1}{2}\left(1+\frac{\varepsilon^{2}}{2}\right)
$$

The pressure rise $(\Delta P)$ is readily found by integrating the pressure gradient w.r.t. $z$ over the interval $[0,1]$ as follows:

$$
\Delta P=\int_{0}^{1} \frac{d p}{d z} d z
$$

The stream function in the wave frame (obeying the Cauchy-Riemann equations, $u=\frac{\partial \psi}{\partial y}$ and $v=-\frac{\partial \psi}{\partial x}$ ) may be computed by using Eqn. (18). Visualization of streamlines is achieved with Mathematica symbolic software. 


\section{NUMERICAL RESULTS AND DISCUSSION}

In this section, the effect of several physical parameters i.e. slip parameter $\left(\alpha_{1}\right)$ and Darcy number $(D a)$ on velocity profile, flow rate, pressure gradient and stream lines are elaborated with the help of Figs. (2-5).

Figs. 2 represent the change in the velocity of the fluid with respect to certain changes in the constraints.

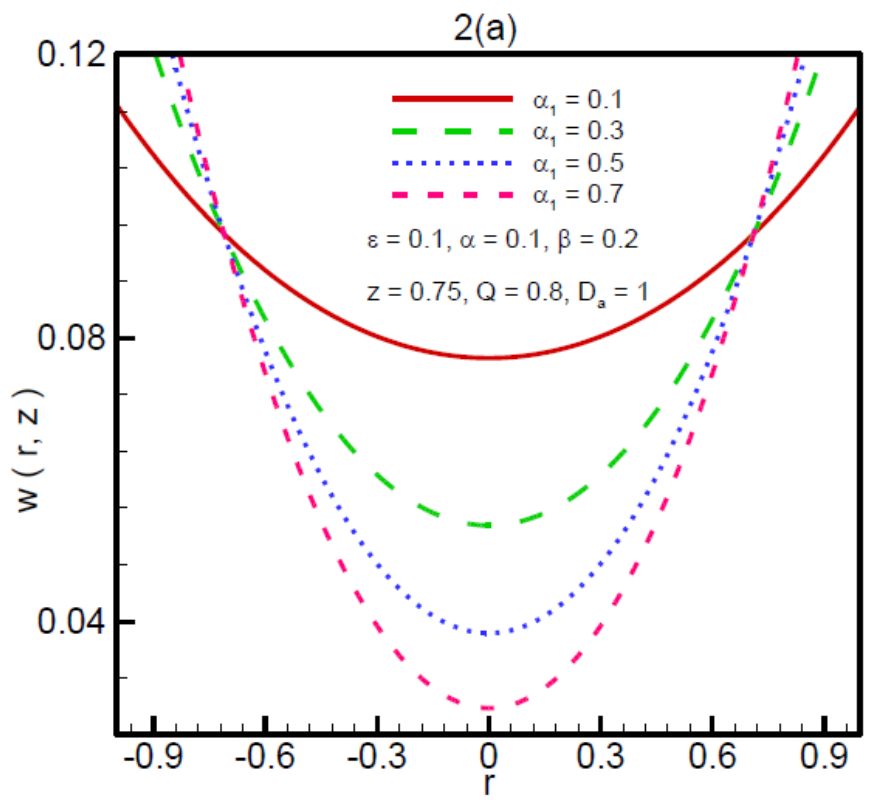



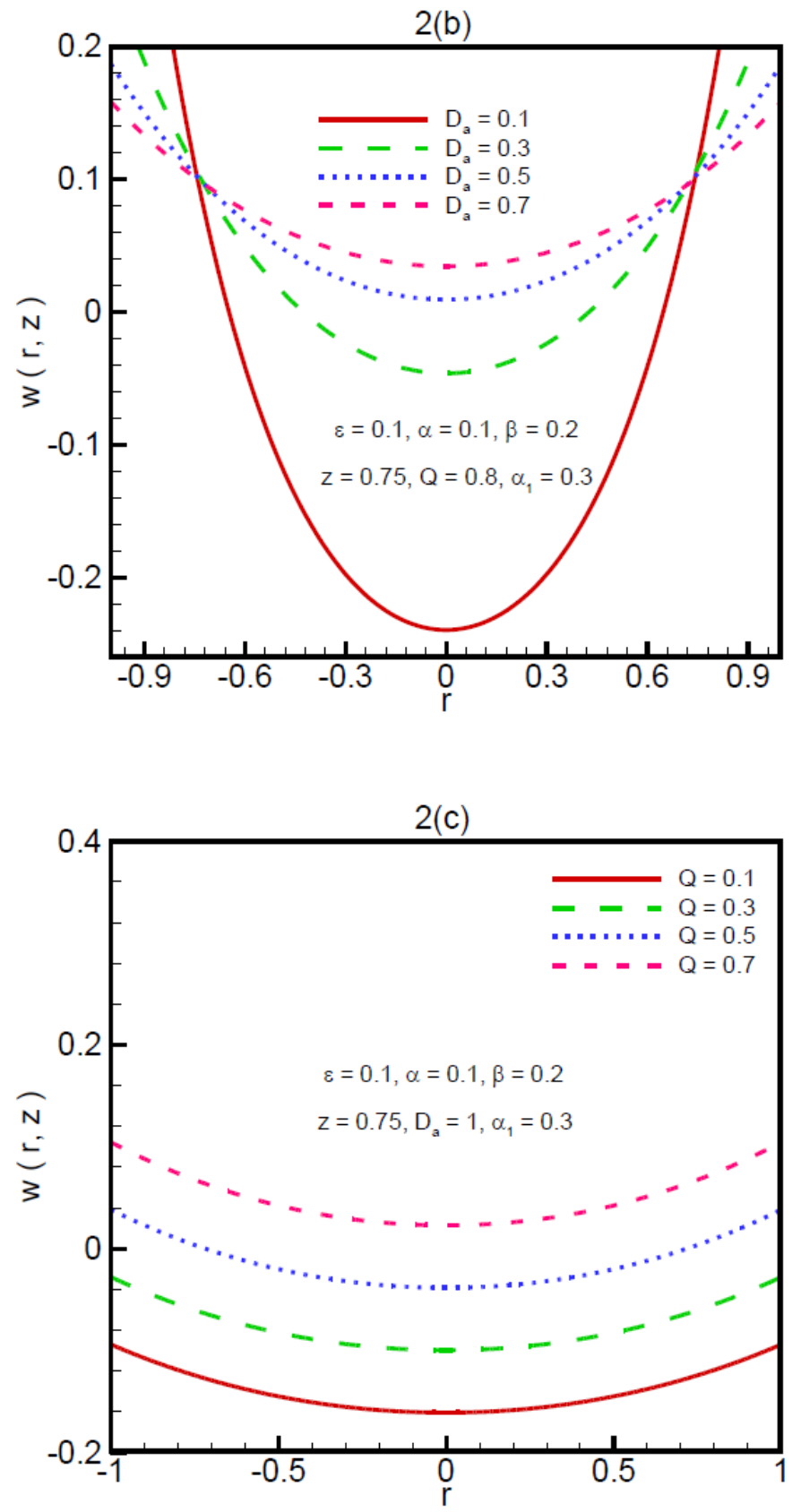


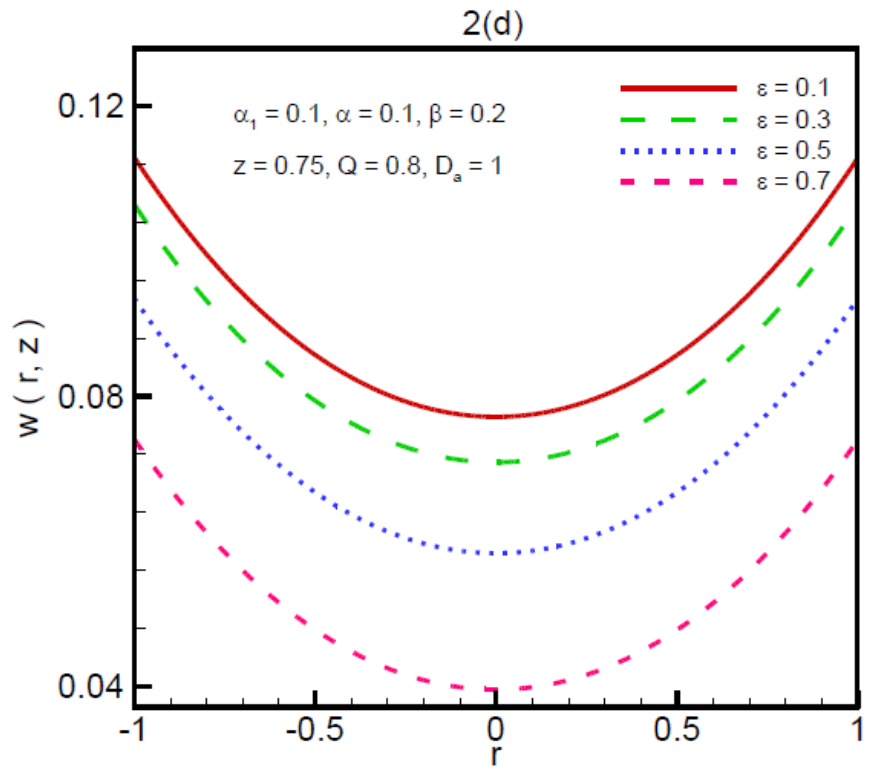

Fig. 2. Velocity profile (axial velocity vs. radial coordinate) for (a) Slip parameter slip parameter $\alpha_{1}$. (b) Darcy number $\mathrm{D}_{\alpha}$. (c) Flow rate Q. (d) Cilia length parameter $\varepsilon$. 

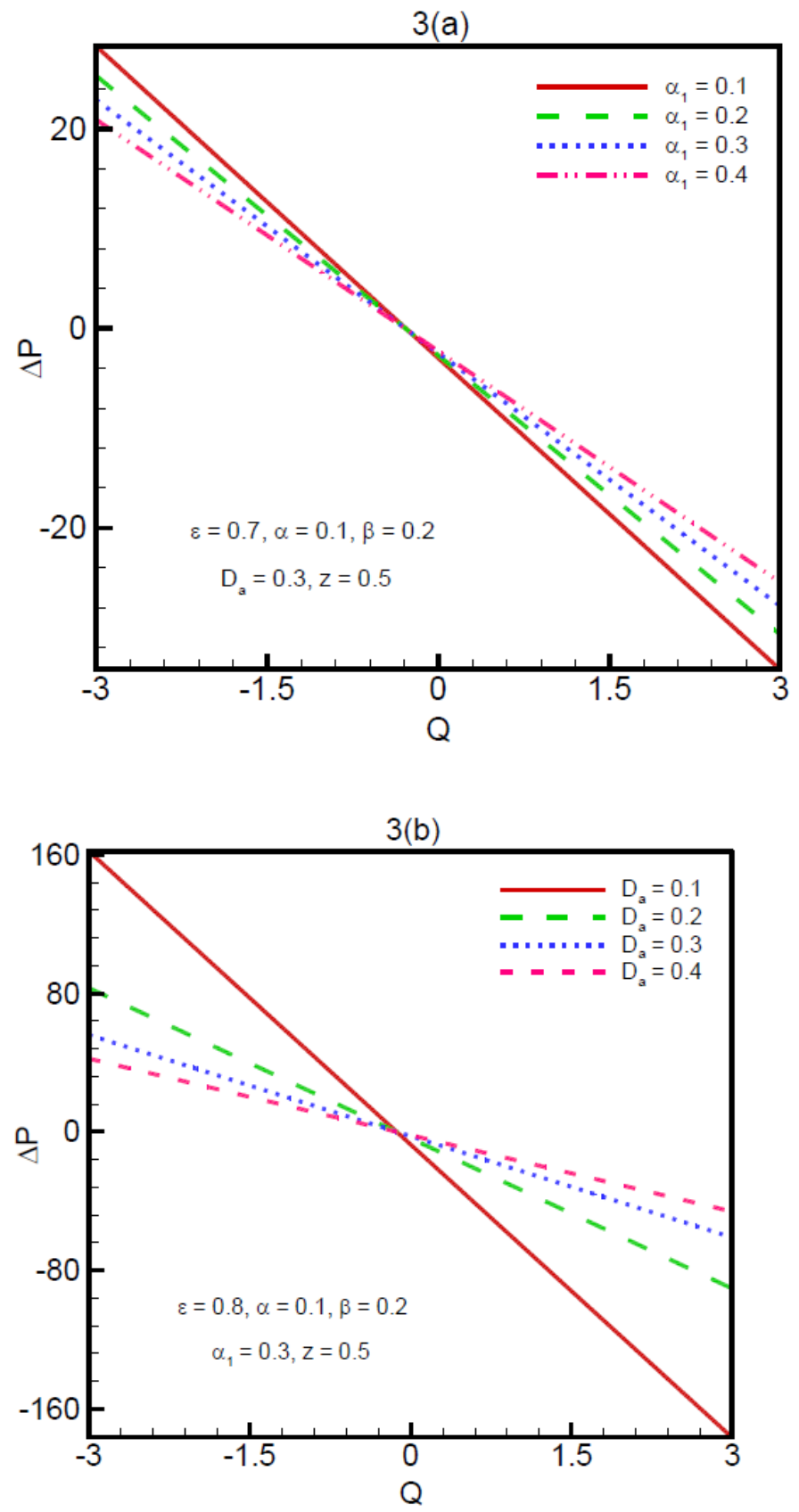


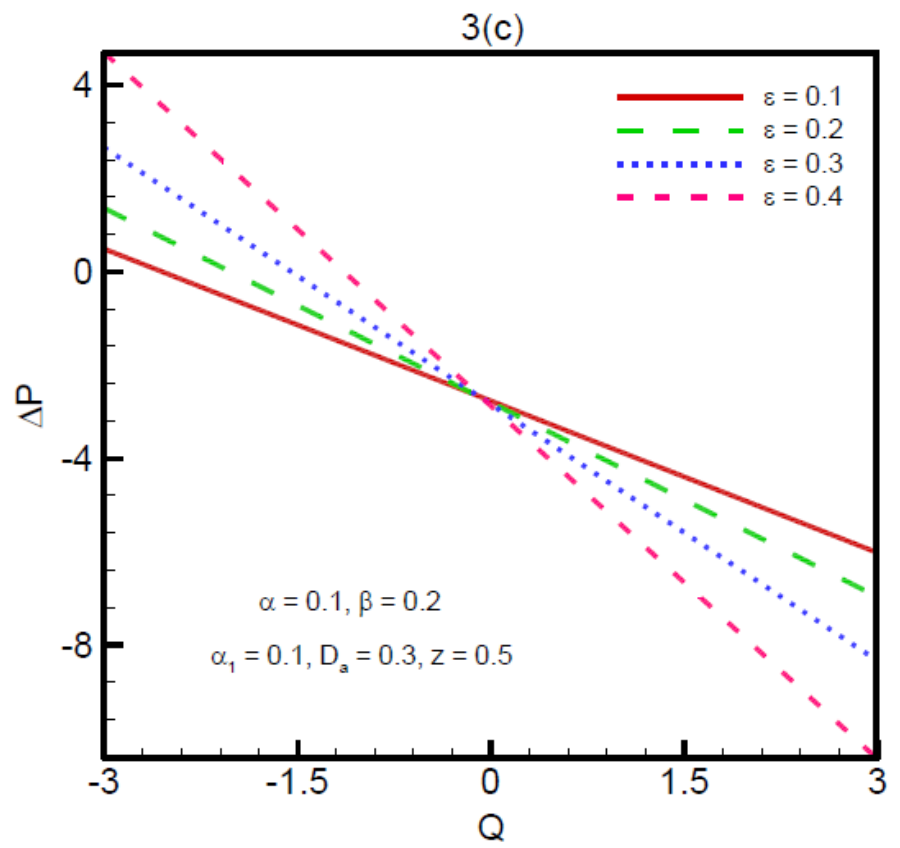

Fig. 3. Pressure rise versus flow rate for (a) Slip parameter slip parameter $\alpha_{1}$. (b) Darcy number $\mathrm{D}_{\alpha} \cdot(\mathrm{c})$ Cilia length parameter $\varepsilon$. 

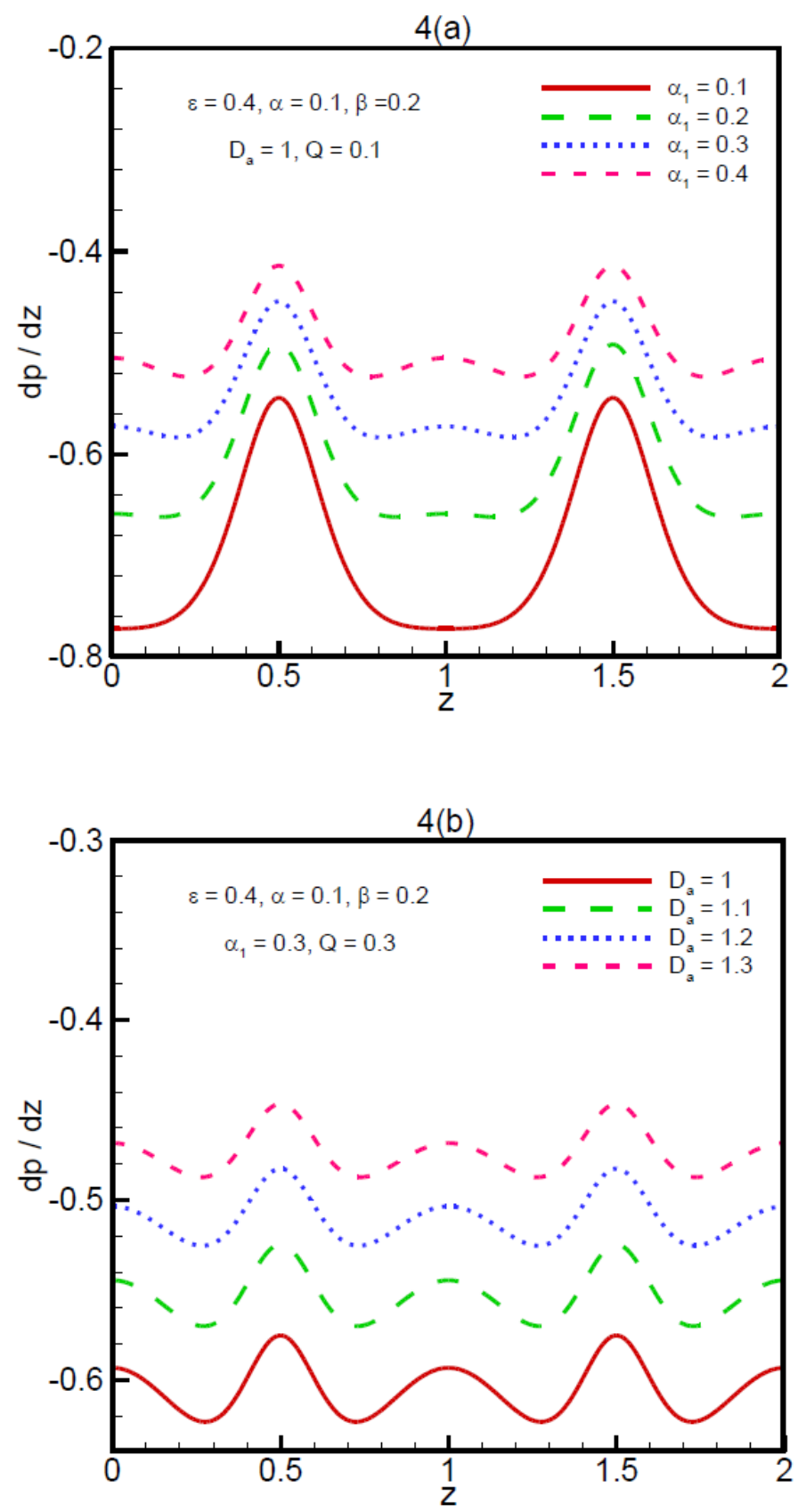


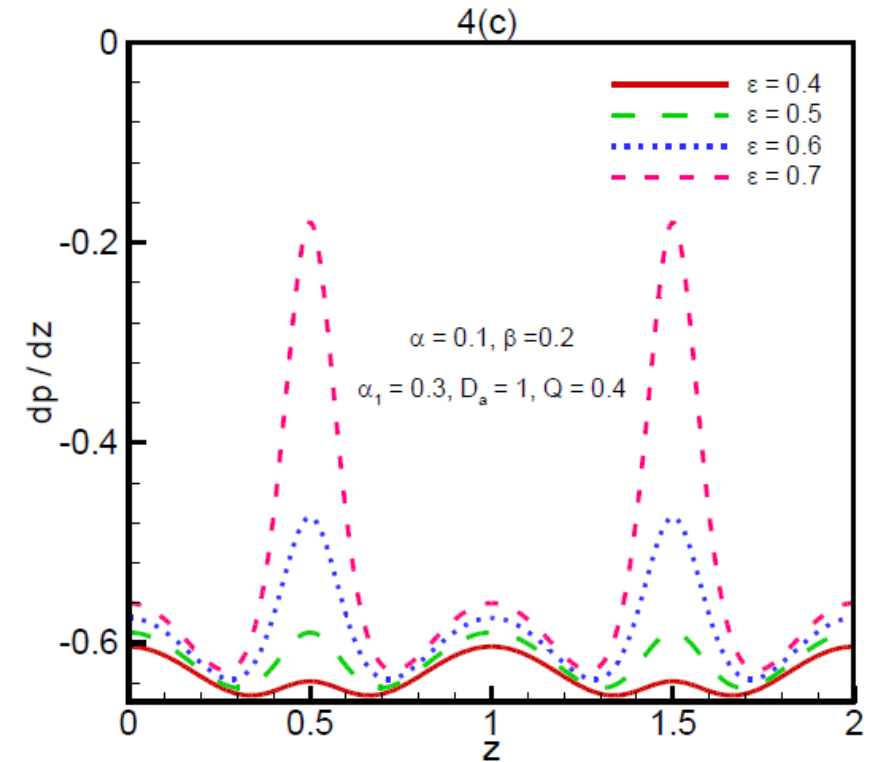

Fig.4. Pressure gradient vs. axial coordinate for (a) Slip parameter slip parameter $\alpha_{1}$. (b) Darcy number $\mathrm{D}_{\alpha}$. (c) Flow rate Q. (d) Cilia length parameter $\varepsilon$. 

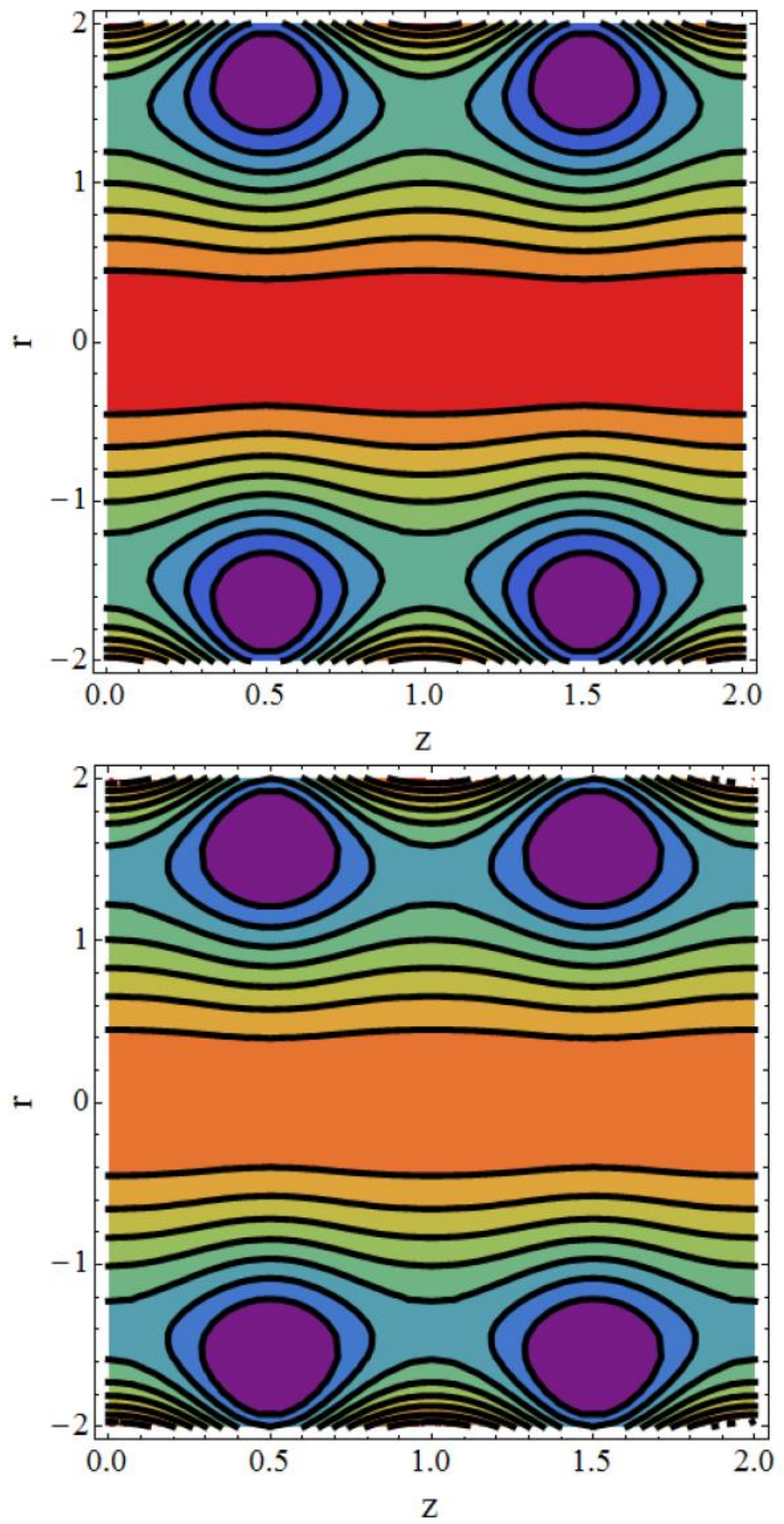

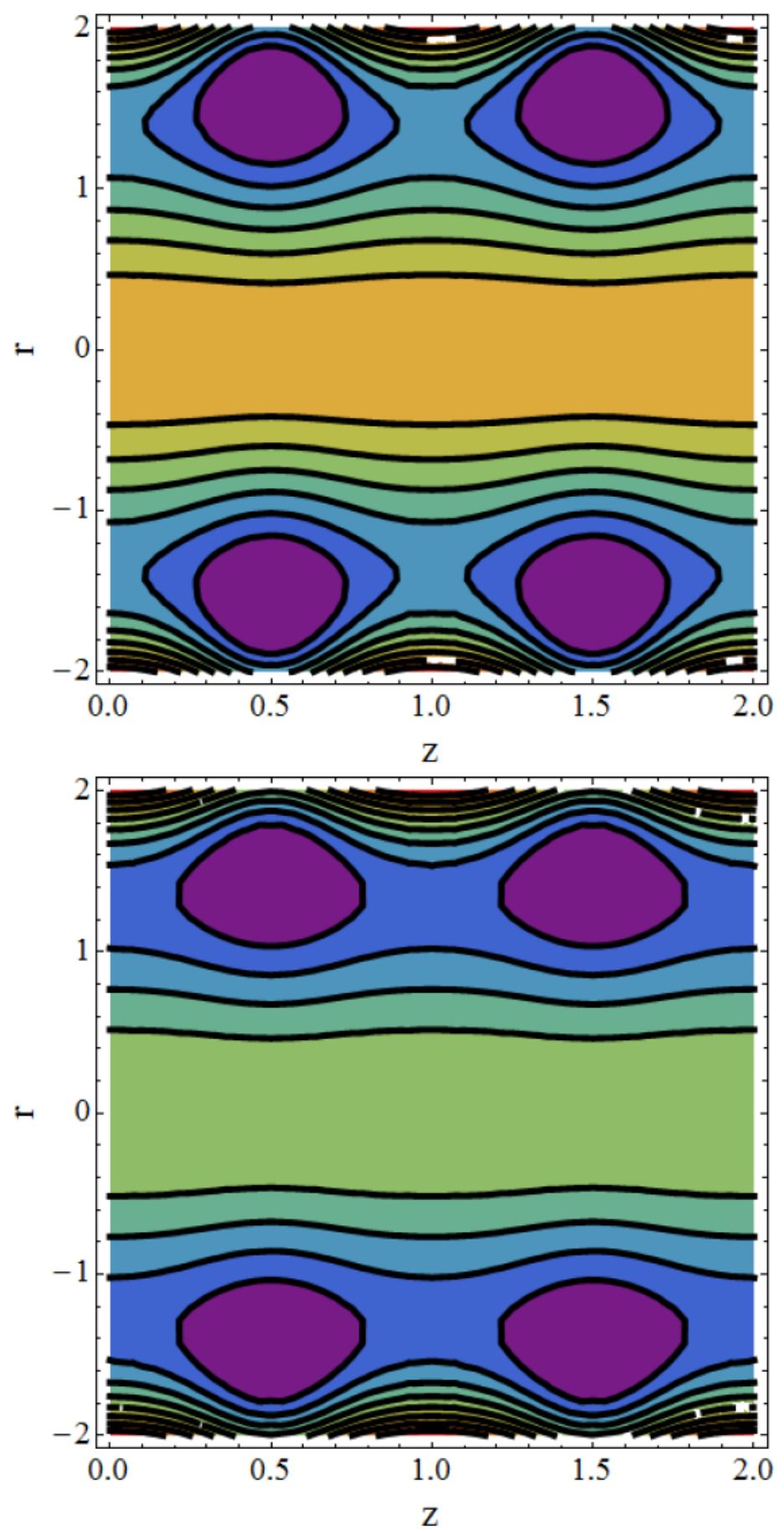

Fig. 5. Streamlines for the velocity profile for slip parameter slip parameter $\alpha_{1}=0.1,0.11,0.12,0.15$ respectively 
The parabolic nature of the velocity profile is clearly evident from these figures. Fig. 2a shows that velocity is inversely proportional to slip parameter $\left(\alpha_{1}\right)$. Greater slip at the wall is found to counteract axial momentum development and manifests in a drop in axial velocity. Fig. $2 \mathrm{~b}$ depicts the effects of Darcy number on velocity profile and it is observed that the velocity is directly proportional to permeability of the medium. Greater permeability corresponds to a significant decrease in Darcian impedance i.e. the drag force associated with solid fibers in the regime. Fig. $2 \mathrm{c}$ represents the influence of flow rate on velocity profile and it is apparent that if flow rate is increasing, velocity also increases. Propulsion is therefore enhanced with greater flow rates. Fig. 2 d reveals that velocity profile diminishes with increasing cilia length parameter (ع). Cilia spacing and length influences the viscous resistance per cilium and thereby the axial flow. The latter is assisted with greater cilia length and this aids in pressure rise in the lower channel half space. The introduction of extra energy to the flow at the lower wall however must be compensated for by an extraction at the upper wall, and these features are also related to synchronicity of beating cilia. The special case of $\varepsilon=0$ implies vanishing cilia and absence of a metchronal wave- in this scenario the flow is a purely peristaltic mechanism due to flexibility of the walls, and is therefore not considered here.

Figs. 3 illustrate the pressure rise evolution with flow rate for various parameters. A linear relation between flow rate and pressure is observed and there are three regions of pumping (i) pumping region $(\Delta P>0)$ (ii) free pumping $(\Delta P=0)$ and (iii) augmented pumping region $(\Delta P<0)$. The effect of slip parameter on pressure rise is shown in Fig.3a and it is evident that pressure diminishes with rise of slip parameter in the pumping region whereas the converse response is computed in the augmented pumping region. The impact of Darcy number on pressure rise is illustrated in fig. $3 \mathrm{~b}$ and exerts a similar effect as with the slip parameter. From fig $3 \mathrm{c}$, it is seen that pressure rise is an increasing function of amplitude in the pumping region whereas it is decreasing function in augmented pumping region.

Figs. 4a-c depict the pressure gradient distributions with axial coordinate for various hydromechanical and geometric parameters. Inspection of the figures reveals that pressure gradient increasing with small changes in slip parameter and Darcy number $\left(\alpha_{1}, D_{a}\right)$ and also 
cilia length parameter $(\varepsilon)$. In all cases, the maxima (peaks) surface at $z=0.5,1.5$, however the relative minima change with modification in parameter values.

Figs 5a-d present the streamline visualizations for the influence of slip parameter. These streamlines constitute a family of curves which are tangential to the velocity vector at any instant of the flow. These show the direction of the fluid element at any point in time. The plots generally demonstrate that with increasing the magnitude of slip parameter $\left(\alpha_{1}\right)$, the trapped bolus inside the streamlines increases in size whereas the number of bolus decreases. In much of a digestive tract such as the human gastrointestinal tract, smooth muscle tissue contracts in sequence to produce a peristaltic wave, which propels a ball of food (called a bolus while in the esophagus and upper gastrointestinal tract and chyme in the stomach. So trapped bolus are enclosed streamlines. From the graphs we can see that near the tube walls more trapped bolus occurs because there is some fluid resistance and at center fluid moves easily and free stream occurs at the center of the tube. So that's why at the center of the tube no tapped bolus occurs all the bolus are near the walls of the tube.

Evidently in summary the geometric and hydrodynamic parameters result in non-trivial modifications in the propulsion characteristics and these warrant further exploration in the future.

\section{CONCLUDING REMARKS}

In this paper, a mathematical study has been conducted for creeping flow induced by a metachronal wave generated by beating of cilia tips in viscous propulsion through a porous medium-filled tube. The study has been motivated by further expounding biological propulsion mechanisms e.g. motion induced by cilia tips in uro-dynamics, breathing in the human respiratory system, sperm movement in reproductive system. Furthermore the study is pertinent to biomimetic propulsion mechanisms exploiting surface treatments via artificial cilia. The work it is envisaged will further stimulate much-needed laboratory investigations which would serve to garner vital experimental data. Although confined to viscous (Navier-Stokes) fluids and viscous-dominated porous media transport (Darcy model), some useful deductions can be made from the computations, namely:

1. Velocity is a decreasing function of slip parameter whereas it is an increasing function of Darcy number. 
2. Greater flow rate elevates axial velocity whereas the latter decreases with increasing axial coordinate.

3. Pressure rise reduces with increasing the effects of slip velocity and permeability (higher Darcy number) in the pumping region whereas the reverse trend is observed in the augmented pumping region.

4. Axial coordinate and Darcy number exert the opposite effect on the pressure rise.

5. The size of trapped bolus of stream line increases with increasing the magnitude of slip parameter.

Acknowledgement:

Authors are thankful to the reviewer for useful suggestion to improve the manuscript.

\section{REFERENCES}

[1] T.Y. Wu, On theoretical modelling of aquatic and aerial animal locomotion, Adv. Appl. Mech., 38, 291-353 (2001).

[2] Wu, T.Y. Reflections for resolution to some recent studies on fluid mechanics. In Advances in Engineering Mechanics - Reflections and Outlooks, World Scientific, Singapore (2006).

[3] J. Feng and S. K. Cho, Mini and micro propulsion for medical swimmers, Micromachines, 5, 97-113 (2014).

[4] Ye Wang, Yang Gao, Hans M. Wyss, Artificial cilia fabricated using magnetic fiber drawing generate substantial fluid flow, Microfluid Nanofluid (2015) 18:167-174.

[5] M. A. Sleigh, The Biology of Cilia and Flagella, MacMillan, New York, USA(1962).

[6] T. J. Lardner, W. J. Shack, Cilia Transport. Bull Math Biophys, 34 (1972) 25-35.

[7] J. R. Blake, A model for the micro-structure in ciliated organisms, J. Fluid Mech. 55 (1972) $1-23$.

[8] T. Y. Wu. Fluid mechanics of ciliary propulsion. Proc. Tenth Annual Meeting of the Society of Engineering Science, Yale University, Connecticut, USA (1973).

[9] C. Brennen, An oscillating-boundary-layer theory for ciliary propulsion. J. Fluid Mech., 65 (1974) 799-824.

[10] M. A. Sleigh and E. Aiello. The movement of water by cilia. Acta. Protozool., 11 (1972) 265-277.

[11] H. Agarwal, A. Uddin, Cilia transport of bio-fluid with variable viscosity, Indian J Pure 
Appl Math., 15 (1984) 1128-1139.

[12] J. R. Blake, A spherical envelope approach to ciliary propulsion, J. Fluid Mechanics., 46 (1971) 199-208.

[13] C.E. Miller, An investigation of the movement of Newtonian liquids initiated and sustained by the oscillation of mechanical cilia, Aspen Emphysema Conf., Aspen, Colorado, USA (1967).

[14] C. Barton, S. Raynor Analytical investigation of cilia induced mucus flow, Bull Math. Biophysics., 29 (1967) 419-428.

[15] D.J. Smith, E. A. Gaffney, J.R. Blake, A viscoelastic traction layer model of muco-ciliary transport, Bull. Mathematical Biology, 69 (2007) 289-307.

[16] A. Dauptain, J. Favier, A. Battaro, Hydrodynamics of ciliary propulsion, J. Fluids and Structures, 24 (2008) 1156-1165.

[17] S. N. Khaderi, P. R. Onck, Fluid-structure interaction of three-dimensional magnetic artificial cilia, J. Fluid Mechanics, 708 (2012) 303-328.

[18] S. N. Khaderi, J. M. J. den Toonder and P. R. Onck, Fluid flow due to collective nonreciprocal motion of symmetrically-beating artificial cilia, Biomicrofluidics., 6 (2012) 014106. [19] S. N. Khaderi, C. B. Craus, J. Hussong, N. Schorr,d J. Belardi, J. Westerweel, O. Prucker,d J. Ruhe, J. M. J. den Toondere and P. R. Onck, Magnetically-actuated artificial cilia for microfluidic propulsion, Lab Chip, 11 (2011) 2002.

[20] G.A. Truskey, F. Yuan and D.F. Katz, Transport Phenomena in Biological Systems, Pearson, New Jersey (2004).

[21] K. Vafai, Handbook of Porous Media, Begell House, New York, USA (2003).

[22] O. Coussy, Mechanics of Porous Continua, Butterworths, USA (1993).

[23] A.R.A. Khaled and K. Vafai, The role of porous media in modeling flow and heat transfer in biological tissues, Int. J. Heat and Mass Transfer, 46 (2003) 4989-5003.

[24] P. G. Saffman, On the boundary condition at the interface of a porous medium, Stud. Appl. Math., 50 (1971) 93-101.

[25] Y. Miyamoto, M. Hanano and T. Iga, Concentration profile in the intestinal tract and drug absorption model: two-dimensional laminar flow in a circular porous tube, J. Theor. Biol. 102 (1983) 585-601.

[26] B. Jeffrey, H.S. Udaykumar and K.S. Schulze, Flow fields generated by peristaltic reflex in 
isolated guinea pig ileum: impact of contraction depth and shoulders, Amer. J. Physiol Gastrointest Liver Physiol 285 (2003) G907-G918.

[27] E.F. Elshehawey, N.T. Eldabe, E.M. Elghazy and A. Ebaid, Peristaltic transport in an asymmetric channel through a porous medium, Applied Mathematics and Computation 182 (2006) $140-150$.

[28] A.R. Rao and M. Mishra, Peristaltic transport of a power-law fluid in a porous tube, J. NonNewtonian Fluid Mech. 121 (2004) 163-174.

[29] D. Tripathi and O. Anwar Bég, Magnetohydrodynamic peristaltic flow of a couple stress fluid through coaxial channels containing a porous medium, Journal Mechanics in Medicine and Biology, doi: 10.1142/S0219519412500881 (2012).

[30] D. Tripathi and O. Anwar Bég, A numerical study of oscillating peristaltic flow of generalized Maxwell viscoelastic fluids through a porous medium, Transport in Porous Media, 95, 337-348 (2012).

[31] R. Ellahi, S. U. Rahman, S. Nadeem and Noreen Sher Akbar, Influence of Heat and Mass Transfer on Micropolar Fluid of Blood Flow Through a Tapered Stenosed Arteries with Permeable Walls, Journal of computational and theoretical nanosciences, 11(2014) 1156-1163.

[32]. S. Nadeem and H. Sadaf, Theoretical analysis of Cu-blood nanofluid for metachronal wave of cilia motion in a curved channel. Transaction on Nanobiosciences, 14(2015)447-454.

[33]. Noreen Sher Akbar,Z. H. Khan, Heat transfer analysis of bi-viscous ciliary motion fluid, Int. J. Biomathematics, 2015 Vol. 8, No. 2 (2015) 1550026 (13 pages).

[34]. Noreen Sher Akbar, Biomathematical analysis of carbon nanotubes due to ciliary motion, Int. J. Biomathematics, Vol. 8, No. 2 (2015) 1550023 (11 pages).

[35]. S. Nadeem, H. Sadaf, Ciliary motion phenomenon of viscous nanofluid in a curved channel with wall properties, European Physical Journal - Plus (EPJ Plus) (2016), 131:65 